\title{
Prestack Full-Waveform Elastic Inversion versus Linearized AVO Inversion: Real Data Example from the North Viking Open Data Set
}

Sérgio Adriano Moura Oliveira - LENEP/UENF, INCT-GP and Invision Geophysics

Anderson Wilson Peixoto de Franco - Invision Geophysics

Uilli Oliveira Freitas - Invision Geophysics

Copyright 2019, SBGf - Sociedade Brasileira de Geofísica

This paper was prepared for presentation during the $16^{\text {th }}$ International Congress of the Brazilian Geophysical Society held in Rio de Janeiro, Brazil, 19-22 August 2019.

Contents of this paper were reviewed by the Technical Committee of the $16^{\text {th }}$ International Congress of the Brazilian Geophysical Society and do not necessarily represent any position of the SBGf, its officers or members. Electronic reproduction or storage of any part of this paper for commercial purposes without the written consent of the Brazilian Geophysical Society is prohibited.

\begin{abstract}
Currently, elastic inversion methods used in E\&P industry are based in linearized approximation for the $P$ wave reflection coefficient. These methods has proved robust for $\mathrm{P}$ impedance estimation, however it is not suitable for estimating parameters that need angles of incidence above 30 degrees in order to be determined. For such parameters, as $\mathrm{S}$ wave velocity or density, full wave form inversion is a more appropriate method. In this work this is demonstrated through a geophysical reservoir characterization case study using the North Viking Graben open data set.
\end{abstract}

\section{Introduction}

When using angles greater than $30^{\circ}$, conventional AVA/AVO methods, using linearized expressions, usually have two fundamental restrictions: reliability of the computed reflection coefficient, limited by the critical angle; and incomplete modeling of wave propagation in layered media to account for converted waves, internal multiples and transmission effects. These events cause the AVA curve to deviate from the behavior predicted by the modeling procedure that only takes primary reflections into consideration (Mallick, 2007).

This effect is accentuated for large angles, especially in the presence of thin layers with strong contrasts in elastic properties, as demonstrated by Simmons and Backus (1994) who pointed out that the locally converted shear waves often have a first order effect in the AVA response, a fact that has not been widely recognized until now. The same phenomena were confirmed by Assis et al. (2016, 2017) based on a reduced scale seismic physical modeling experiment. Another problem is that the conventional AVO method overestimates the data sensitivity, in relation to $\mathrm{Vp}$ and $\mathrm{Vs}$, for angles above 30 degrees (Oliveira et all, 2018).

The consequence of not taking into account the locally converted waves on elastic inversions can be significant, as pointed out by Hounie and Oliveira (2014), whose conclusion is supported by an investigation involving synthetic data. In this work we show that this fact is also confirmed in a real data example using the the North
Viking Graben open data set were we compare linearized AVO inversion based on Aki and Richards approximation and a prestack elastic FWI method.

\section{Method}

Linear AVA inversion is based on approximations of the Zoeppritz equation solution, represented in terms of contrasts between elastic properties in geologic layers (Aki and Richards, 1980; Fatti et all, 1994). Approximations for the reflection coefficient of $\mathrm{P}$-waves, known as the three-term expression, can be generically written as

$$
R_{p p} \cong a r_{\alpha}+b r_{\beta}+c r_{\rho}
$$

where $r_{\alpha}=\left(\alpha_{2}-\alpha_{1}\right) /\left(\alpha_{2}+\alpha_{1}\right), r_{\beta}=\left(\beta_{2}-\beta_{1}\right) /\left(\beta_{2}+\beta_{1}\right), r_{\rho}=$ $\left(\rho_{2}-\rho_{1}\right) /\left(\rho_{2}+\rho_{1}\right)$ and coefficients $a, b$, and $c$ depend on the angle of incidence and the ratio between $S$ and $P$ wave velocities. This can be represented in the following matrix equation for modeling amplitudes as a function of angle:

$$
\mathrm{Mr}=\mathbf{d}
$$

where $\mathbf{M}$ is a matrix of coefficients with three columns and $\mathrm{N}$ rows: $\mathbf{M}(N \times 3)$ whose elements are given by $m_{i, 1}=a_{i}$, $m_{i, 2}=b_{i}$, and $m_{i, 3}=c_{i}$. Coefficients of the column vector $\mathbf{r}$ are given by $r_{\alpha}, r_{\beta}$, and $r_{\rho}$, $\mathbf{d}$ is the vector whose elements are reflection amplitudes for the incidence angle $\theta_{i}$ and $N$ is the number of angles. In AVA inversion, the vector d contains reflection amplitudes for different angles. The system represented by equation 2 can be solved by the least-squares method for determining $\mathbf{r}$, leading to the following normal equation (Menke,1989):

$$
\mathbf{M}^{\mathrm{T}} \mathbf{M r}=\mathbf{M}^{\mathrm{T}} \mathbf{d} .
$$

This is the simplest and most direct type of elastic inversion. It determines $r_{\alpha}, r_{\beta}$, and $r_{\rho}$ for each temporal sample in a set of traces ordered by incidence angle. These schemes also assume that the seismic data only contain primary reflections and ignore the transmission effect within layers.

Elastic full waveform inversion is based on complete elastic seismic forward modeling, so it is necessary to consider a nonlinear mathematical relationship between the data and model parameters:

$$
\mathbf{d}=\mathbf{S}(\mathbf{m})
$$


where $\mathbf{S}$ stands for the mathematical operator that connects the data vector $\mathbf{d}$ to the model parameter vector $\mathbf{m}$. In this study, the subsurface is represented as a 1D geological medium divided into elemental layers with constant thickness. The elastic full waveform inversion method we used is named AVA-FWI. This method uses optimized computational routines based on the reflectivity method for efficient calculation of synthetic angle gather and differential angle gather (Oliveira et all, 2018).

The inversion is conducted by a nonlinear data fitting, implying that the solution is given by a vector $\mathbf{m}$ that minimizes an error function that measures the distance between the observed and calculated data. We choose the L2 norm criterion which yields:

$$
E(\mathbf{m})=\frac{1}{2} \Delta \mathbf{d}^{\mathrm{T}} \Delta \mathbf{d}
$$

where $\mathbf{\Delta} \mathbf{d}=(\mathbf{d}-\mathbf{S}(\mathbf{m}))$ is a vector whose elements are the difference between the calculated and observed data. The inverse problem allows several solutions that meet a given tolerance criteria to minimize the objective function. This happens because the seismic data contain noise, and have limited spatial coverage and frequency content. The presence of noise and the incomplete nature of this data make it impossible to recover the exact elastic parameter model from inversion. Physically, seismic waveform inversion can only recover the model features that are, in terms of dimension, compatible with the propagated wavelengths.

The input data for AVA-FWI are seismic traces from prestack, time migrated common reflection point gathers and ordered by angle of incidence. These data have more favorable signal-to-noise ratio, especially after it has been submitted to preconditioning routines (Braga and Moraes, 2013). Another advantage is that migration collapses diffractions and corrects for the effects of dip and curvature of the reflectors. Therefore, we can address the subsurface as locally $1 \mathrm{D}$.

\section{Example}

The real data used in this work comes from North Viking Graben in the North Sea. It is an open data set made available for the purpose of testing seismic inversion methods and amplitude preserving seismic processing work flows (Keys and Foster, 1998). It contains a 2-D unprocessed seismic line (25 km long) and two wells having $\mathrm{P}$-wave velocity, density and S-wave velocity. The complete data set also contains a far field wavelet. The seismic data have been pre-processed and migrated, using an amplitude preserving PSTM working flow. The image gathers are further subjected to a preconditioning with the objective of residual multiples elimination and residual move out correction. The preconditioned image gathers were transformed to the angle domain by an angle stack routine. The angle range used for inversion was five to forty degrees.
The main objectives for this study are the clastic reservoirs of Jurassic age that occur below 2 seconds in well $A$. The reservoirs occur in Jurassic syn-rift sediments and are vertically stacked and separated by deep water shale. They can be associated with stratigraphy truncation at the top of Jurassic although some are faulted bounded. The inversion was restricted to a time interval around well $A$, where three reservoir levels occur, as indicated in Figure 1. The presence of hydrocarbons has a large impact recognized as a decrease in the density. The hydrocarbons have little effect on the P-wave velocity and a very little effect in S-wave velocity.

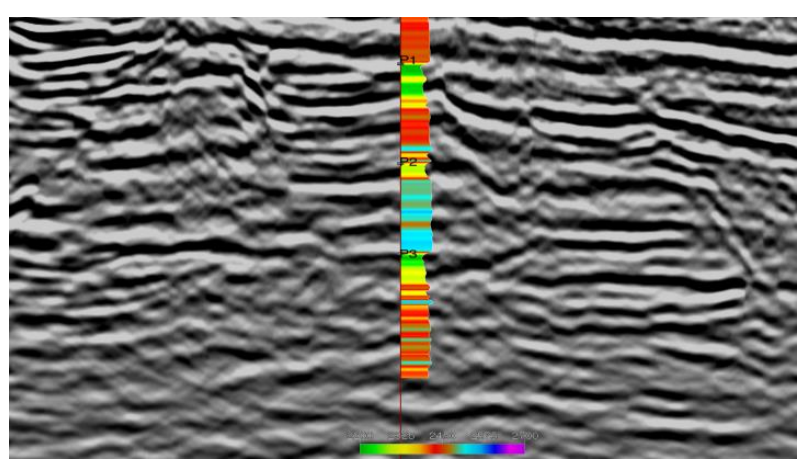

Figure 1 - Expanded view of the study area in the final migrated section of the North Viking line. The three reservoir top levels are marked in the inserted density log.

The seismic data has a frequency bandwidth of 6 to 75 $\mathrm{Hz}$, so information regarding the models low frequency trends can't be estimated from this signal. The initial models for inversion of $\mathrm{Vp}, \mathrm{Vs}$ and density are obtained by extrapolation of these parameters from well logs and following the seismic horizons.

\section{Results}

We begin with the results obtained by AVA-FWI. Notice that the three reservoir levels corresponding to low density layers are clearly seen on the density inversion result (Figure 2). The top of the first reservoir, in the density section, coincides very well with the base of the Cretaceous (white line), which is geologically coherent. The reservoirs layers are also well distinguished in the derived $\mathrm{P}$-wave impedance section (Figure 3 ) but the first one is not so well delineated in the Vp section, what is predicted by the well log information. The $\mathrm{Vs}$, as expected, is the least sensitive parameter with respect the presence of hydrocarbons and the reservoir layers can't be distinguished in the Vs section. The presence of faults is very visible in the AVA-FWI inverted sections, although they are more evident in the density and in the derived $P$ impedance section. Some of these faults are indicated in the inversion results.

The Aki\&Richards based conventional AVO inversion generated $\mathrm{Vp}, \mathrm{Vs}$ and density sections (Figure 2) that are clearly inferior in terms of continuity and resolution when 
compared with AVA-FWI. The derived AVO Ip section is also inferior to the Ip section from AVA-FWI (figure 3). Not that the three reservoir levels can be easily identified in the FWI density section, however the second reservoir level is not present in the AVO density section, in addition the third reservoir level are misplaced and the first level, although identifiable, is very poorly defined in the conventional AVO inversion result. It is important to point out that any kind of regularization to promote lateral continuity was used. The inversion of each gather was performed independently of the others in both methods.

\section{Conclusions}

Conventional AVA/AVO methods, using linearized expressions, have serious restrictions like reliability of the computed reflection coefficient, limited by the critical angle; and incomplete modeling of wave propagation in layered media. AVA-FWI doesn't suffer this limitations and it can estimate the density in a robust way, together with $\mathrm{Vp}$ and $\mathrm{Vs}$, provided the input data has an angle range greater than 30 degrees. This was fully confirmed is this study where both methods were used to invert the North Viking open data set. The Vp, Vs and specially the density section produced by Linearized AVO inversion method is quantitatively inferior and also inferior in terms of continuity and resolution than the results from AVAFWI.

\section{Acknowledgments}

We thank Sinochem Brazil for financial support and Invision Geophysics for the facilities to run the inversion tests.

\section{References}

Aki, K., and P. G. Richards, 2002. Quantitative Seismology. University Science Books 2nd ed.

Assis, C. A. M, S. A. M. Oliveira, R. M. Missagia and M. A. R. Ceia, 2016. The importance of locally converted shear waves in the thin layers AVO response - A physical modeling study. $78^{\text {th }}$ EAGE Conference and Exhibition expanded abstracts.

Assis, C. A. M, S. A. M. Oliveira, R. M. Missagia and M. A. R. Ceia, 2017. Source wavelet and local wave propagation effects on the amplitude-variation-with-offset response of thin-layer models: A physical modeling study. Geophysics, 82, N27-N41.

Braga, I. L.S., and F. S.Moraes, 2013. High-resolution gathers by inverse $Q$ filtering in the wavelet domain: Geophysics, 78, V53-V61.

Fatti, J. L., G. C. Smith, P. J. Vail, P. J. Strauss and P. R. Levitt, 1994. Detection of gas in sandstone reservoirs using AVO analysis: Geophysics, 59 , 1362-1376.
Hounie, J. N., and S. A. M. Oliveira, 2014. The importance of locally converted waves on elastic inversion of compressional data: Brazilian Geophysical Journal, 32, 323-346.

Keys, R. G., and D. J. Foster, 1998, Comparison of seismic inversion methods on a single real data set, Open file publications no. 4: SEG.

Mallick, S., 2007. Amplitude variation with offset, elastic impedance, and wave-equation synthetics - A modeling study: Geophysics, 72, C1-C7.

Menke, W., 1989. Geophysical data analysis: Discrete inverse theory. Academic Press, San Diego.

Oliveira, S. A. M., I. L. S. Braga, M. B. Lacerda, G. F. Ouverney and A. W. P. de Franco, 2018. Extending the usefull angle range for elastic inversion through the amplitude versus angle full-waveform inversion method. Geophysics, 83, R213-R226.

Simmons, L. J., and Backus, M., 1994. AVO modeling and the locally converted shear wave: Geophysics, 12371248. 

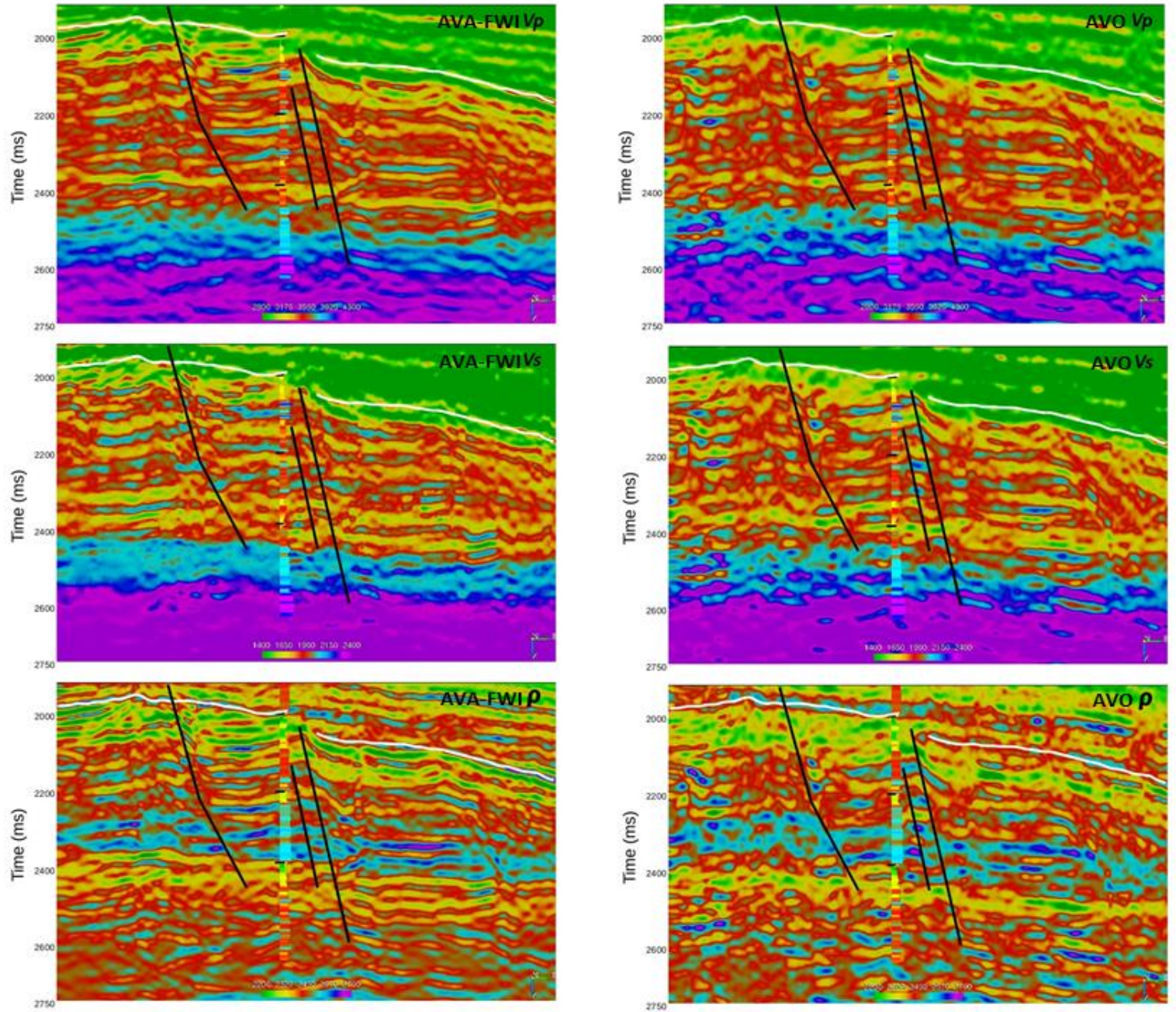

Figure 2 - Vp, Vs and density sections obtained from AVA-FWI and linearized AVO inversion.
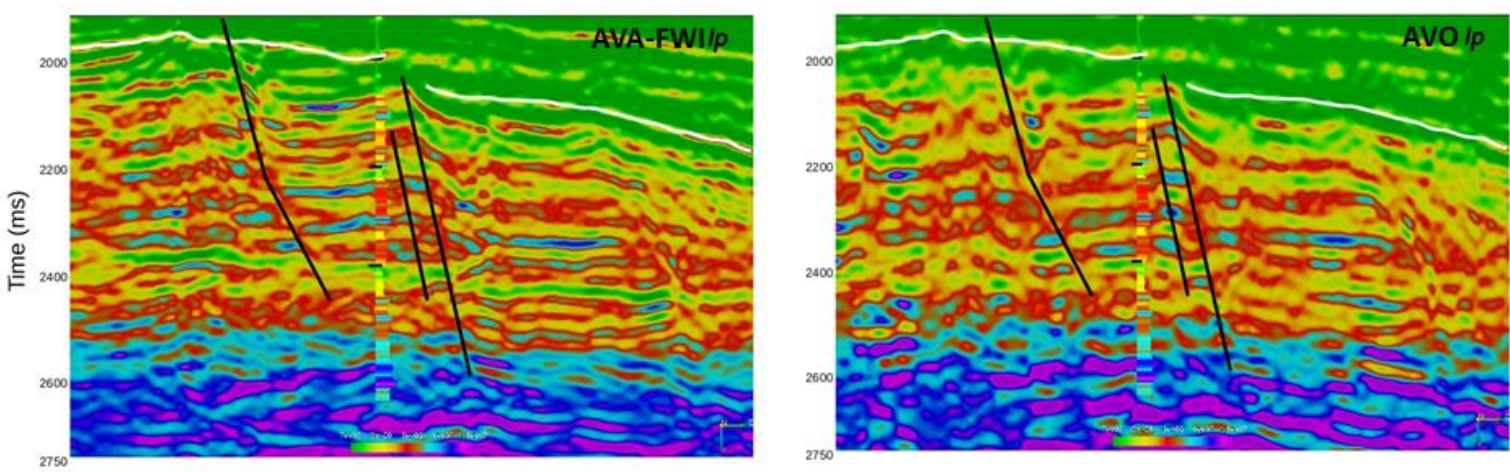

Figure 3 - Derived P wave impedance sections obtained from AVA-FWI and linearized AVO inversion. 